\title{
Editorial
}

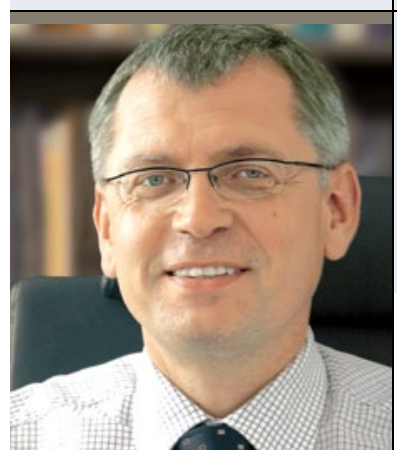

„Es wird immer noch die Mär verbreitet, die Leistungsfähig-

keit des medizinischen Systems, dessen Kern nun einmal

die Ärzte ausmachen, sei immer weiter steigerbar."

\section{Im Arztberuf muss wieder mehr ärztliche Tätigkeit stecken!}

K urz nach der Lektüre des FAZ-Artikels „Der alte Arzt hat ausgedient" (C. Huckenbroich, Frankfurter Allgemeine Zeitung vom 25.4.2012) über geänderte Rahmenbedingungen vieler junger Ärzte hatte ich ein sehr interessantes Vorstellungsgespräch mit einem jungen Kollegen, der seine Präferenzen für eine eventuelle Beschäftigung in unserer Klinik schilderte. In der Zusammenschau habe ich mir einige Gedanken gemacht zur gegenwärtigen Situation der Stellenbesetzung an Krankenhäusern - speziell großen psychiatrischen Kliniken - und wage einige Prognosen für die Zukunft, die ich gern mit Ihnen teilen möchte.

\section{Die Rahmenbedingungen:}

_Es gibt nach wie vor zu wenig Bewerber für freie Arztstellen.

_ Die Zahl der Ärztinnen unter den Bewerbern überwiegt die Zahl der Ärzte deutlich.

_Es besteht seitens der Bewerber ein hoher Bedarf an flexiblen Arbeitszeitmodellen, sowohl was die Wochenarbeitszeit betrifft, als auch die Möglichkeit, zeitweise beurlaubt zu werden, etc.

_ Der Gedanke der Work-Life-Balance hat einen hohen Stellenwert, mit einer zunehmenden Tendenz zugunsten des Bereichs „Life“.

— Ausdrücklicher Wunsch nach wenig Diensten und wenig bis keinen Überstunden.

_Eine möglichst komplette, während der Regelarbeitszeit zu absolvierende Weiterbildung wird als verbindlich eingefordert.

_Es besteht immer weniger Bereitschaft, immer mehr administrative Arbeit neben der eigentlichen ärztlichen Tätigkeit zu übernehmen. Das Ausmaß dieser Arbeit scheint, trotz allseits geäußerter Kritik, in den letzten Jahren weiter zugenommen $\mathrm{zu}$ haben, insbesondere spielen hier die flächendeckende Einführung von Qualitätsmanagement-
Prozessen und die Vorbereitung des neuen Entgeltsystems in der Psychiatrie eine maßgebliche Rolle. Die Hoffnung, dass durch die Einführung elektronischer Werkzeuge, es sei nur das Krankenhausinformationssystem genannt, eine Eindämmung dieser Entwicklung zu erreichen sei, hat sich zerschlagen.

All diese Umstände müssen meines Erachtens dazu führen, dass

_immer weniger Ärzte überhaupt eine Stelle im Krankenhaus antreten beziehungsweise bald nach Arbeitsbeginn wieder aufhören werden und _ die Ärzte im Krankenhaus nicht mehr jede Steigerung der Arbeitsbelastung so stillschweigend tolerieren, wie dies in den vergangenen Jahren oftmals der Fall war.

Gleichzeitig wird allerdings von manchen Kreisen immer noch die Mär verbreitet, die Leistungsfähigkeit des medizinischen Systems, dessen Kern nun einmal die Ärzte ausmachen, sei immer weiter steigerbar, es wird beispielsweise von „Effizienzressourcen“, die es $\mathrm{zu}$ „heben“ gelte, schwadroniert.

\section{Kennziffer: Anteil der ärztlichen Tätigkeit der} Arbeitszeit

Was ist nun also zu tun? Eins ist klar, Sonntagsreden nützen nichts. Sicher werden wir auch nicht umhin kommen, einigen Wünschen der jungen Arztgeneration entgegenzukommen. Der zentrale Punkt ist aber: Wir alle müssen uns auf ärztliche Tätigkeiten rückbesinnen. Wie wäre es zum Beispiel mit einer Kennziffer ganz anderer Art, etwa wie viel Prozent der Arbeitszeit verbringt der Arzt in direktem Patientenkontakt (sollte möglichst hoch sein) und zu wie viel Prozent seiner Arbeitszeit ist er mit administrativen Arbeiten (Dokumentation, Ausfüllen von unterschiedlichsten Erhebungsbögen, Kampf mit der EDV, etc.) beschäftigt (sollte möglichst niedrig sein)? 


\section{Editorial}

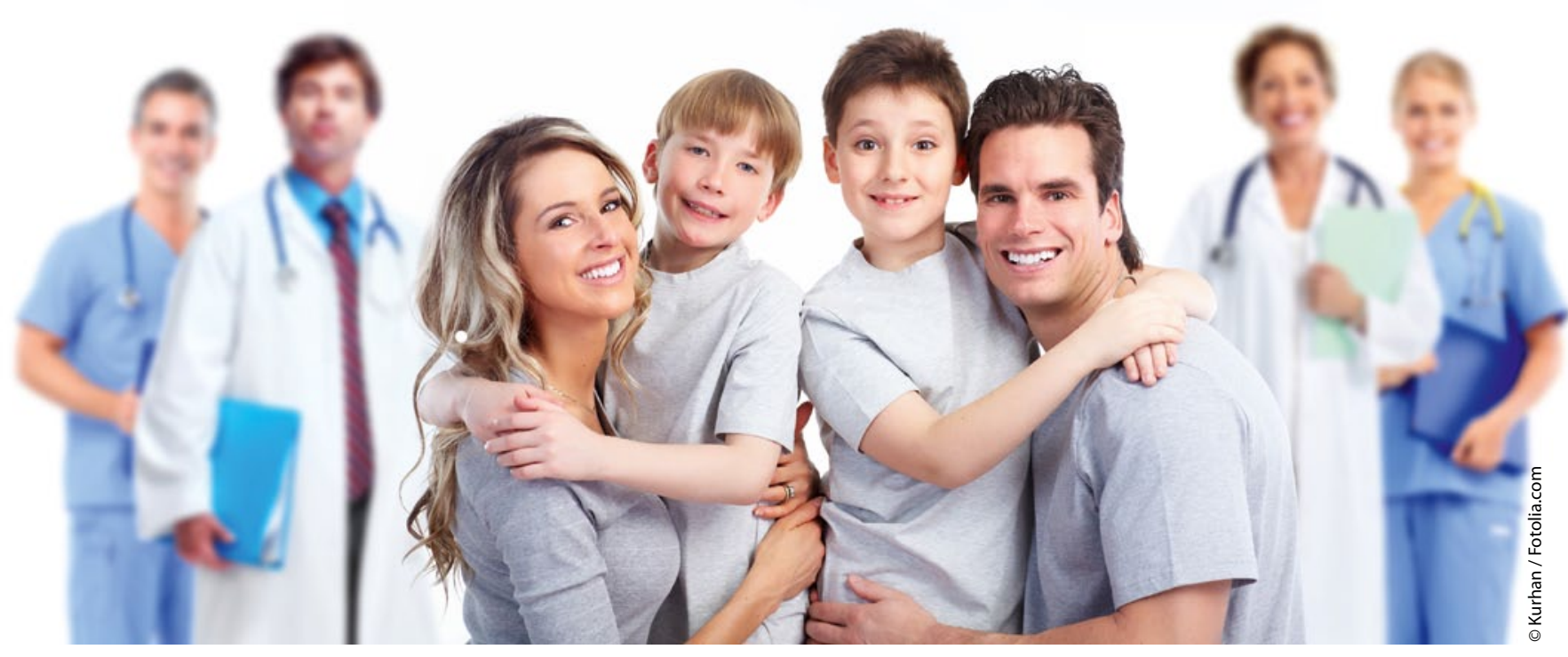

Junge Ärztinnen und Ärzte wissen was sie wollen. Eine ausgewogene Work-Life-Balance gehört auf jeden Fall dazu.

Vielleicht, so meine Hoffnung, würde nämlich die Attraktivität des Arztberufes in Versorgungskrankenhäusern auch dann steigen, wenn der zu erlernende Beruf wieder stärker ärztlich geprägt wäre, frei von dem Ballast, mit dem er über die Jahre immer mehr beschwert worden ist.

Zurück zu der vorgeschlagenen Kennziffer: Es ist schon verwunderlich, dass trotz eines Meers an Kennzahlen, die landauf, landab, immer mit der Begründung, dies müsse unbedingt sein, erhoben werden, solch banalen Dinge wie der Anteil der ärztlichen Tätigkeit an der Arbeitszeit nicht flächendeckend bekannt sind. Wäre das Ergebnis womöglich zu erschreckend? Würde man plötzlich, anstatt der Erhebung noch eines weiteren Surrogatparameters vielleicht der „inneren Qualität“ eines Krankenhauses zu nahe kommen?
Liebe Kolleginnen und Kollegen, nur die Rückbesinnung auf unsere wirklichen ärztlichen Aufgaben werden der Gesellschaft, den Patienten und auch uns nachhaltig helfen!

Mit freundlichen kollegialen Grüßen, Ihr

Hans-Peter Volz

Professor Dr. H.-P. Volz, Ärztlicher Direktor, Krankenhaus für Psychiatrie, Psychotherapie und Psychosomatische Medizin, Schloss Werneck, Balthasar-Neumann-Platz 1, 97440 Werneck

\section{Die Top 3 im Mai auf springermedizin.de}

Dabigatran reduziert tödliche Hirnblutungen

- In der Prophylaxe von Schlaganfällen bei Vorhofflimmern bietet der direkte Thrombinhemmer Dabigatran klinische Vorteile im Vergleich zur oralen Antikoagulation mit Vitamin-K-Antagonisten. $•(2890216)$.

\section{Parkinson lässt sich an den Augen ablesen}

- Patienten mit Morbus Parkinson weisen ein charakteristisches Augenzittern auf, das sich womöglich für die Frühdiagnose der Erkrankung eignet. $>$ (2896290).
Schlaganfallschutz durch Vitamin-K-Antagonisten: heute besser als gestern

- Die Wirksamkeit von Vitamin-K-Antagonisten bei Vorhofflimmern wird durch ältere Studiendaten möglicherweise unterschätzt. Das Schlaganfallrisiko unter Warfarin ist heute deutlich niedriger. (2894814).

- Diese Artikel finden Sie, indem Sie den Titel oder die (in Klammern gesetzte) ID-Nummer in die Suche eingeben. 